\title{
EVALUATION OF HONEYWELL RECOVERABLE COMPUTER SYSTEM (RCS) IN PRESENCE OF ELECTROMAGNETIC EFFECTS
}

\author{
Mahyar Malekpour, NASA Langley Research Center, Hampton, VA \\ Email: m.r.malekpour@larc.nasa.gov
}

\begin{abstract}
The design and development of a Closed-Loop System to study and evaluate the performance of the Honeywell Recoverable Computer System (RCS) in electromagnetic environments (EME) is presented. The development of a Windows-based software package to handle the time critical communication of data and commands between the RCS and flight simulation code in real-time, while meeting the stringent hard deadlines is also presented. The performance results of the RCS while exercising flight control laws under ideal conditions as well as in the presence of electromagnetic fields is also discussed.
\end{abstract}

\section{Introduction}

The problem of verifying the integrity of control computers in adverse as well as nominal operating environments is a key issue in the development, validation, certification, and operation of critical control systems for advanced aircraft. An adverse operating environment of particular concern relative to validation and certification of critical systems is caused by electromagnetic disturbances. Sources of electromagnetic disturbances include lightning, High Intensity Radiated Fields (HIRF) caused by RF transmitters and radars, portable electronics devices carried onto the airplane, and electromagnetic incompatibilities of equipment installed on the aircraft [1].

Soft faults in digital avionics have traditionally been manually corrected. More recently, architecture design measures for the automatic correction of soft faults have begun to be developed. It is perceived that significant benefits can be gained through soft fault protection measures designed into the basic system mechanization. Architecture designs with soft fault protection provide the ability to tolerate disruption of either input/output data or internal computation. Fault clearing and computation recovery must be rapid enough to be "transparent" relative to functional operation and flight deck effect [2].

\section{RCS}

An example of an architectural soft fault protection philosophy in the design of computing platforms is the Aircraft Information Management System (AIMS) used on Boeing 777 aircraft. All computing and I/O management resources are lock-step compared on a processor cycle-by-cycle basis. In this approach, if a soft or hard fault event occurs, the processor is interrupted and service handlers take control and no data can be exported [2].

The first stage of a prototype computing platform for fast recovery from soft faults has been developed for NASA. This prototype includes patented technology for transparent soft fault recovery that had never been built or tested. These concepts were implemented and integrated into the basic lock-step processing module of the AIMS architecture. This prototype was delivered to NASA in 1997 for evaluation at the Langley Research Center (LaRC) system integration facility. A general illustration of a computing platform with rapid (transparent) recovery elements is depicted in Figures 1 and 2. 


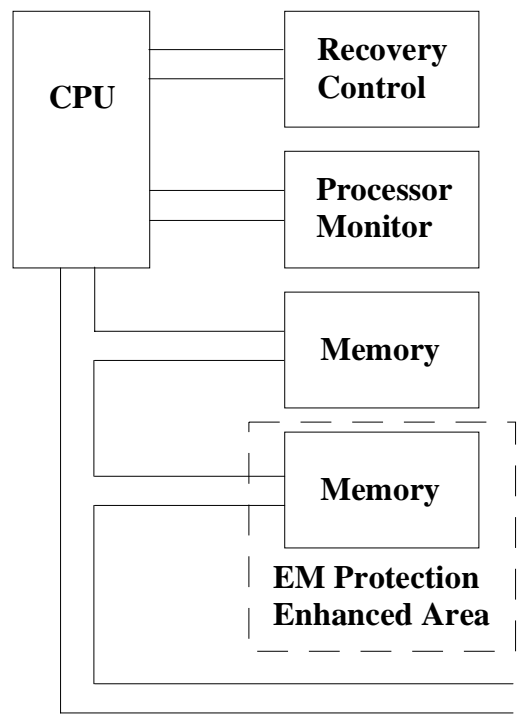

Data Bus

Figure 1. Digital Processor with Elements for Rapid Recovery

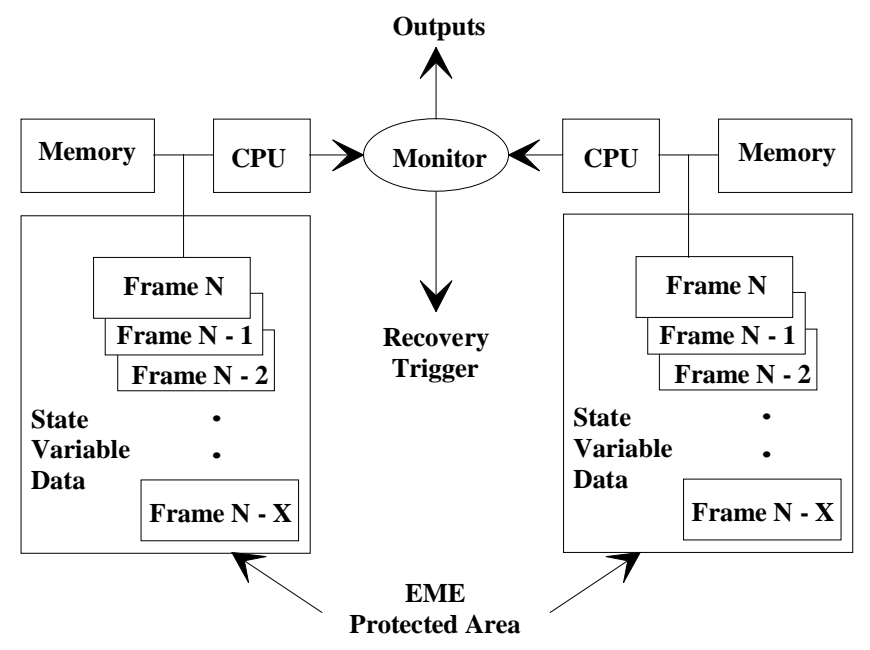

Figure 2. Rapid Recovery Upset Detection and Recovery Flow

Such a platform is intended to provide soft fault recovery that would be virtually software independent and transparent to a system function. As shown in Figure 2, the rapid recovery concept involves protecting and recovering the significant state variables of a system function. An aircraft autoland (arm, capture and track) function is being used for evaluation of the rapid recovery concept. Since this is the only function being implemented in the application program software, the partitioning portion of the platform SAFEbus technology was not employed. Communication with the LaRC host system integration facility is intended to be accomplished primarily through an optical 429 bus structure. LaRC has identified the platform integrated with the host facility as the RCS [2].

\section{System Architecture}

The proposed Closed-Loop architecture consists of the RCS, a VME-based 429-optic card for conversion of data from electrical to optical and vice versa, a flight simulation host processor, and a PC for the development and display of data as shown in Figure 3.

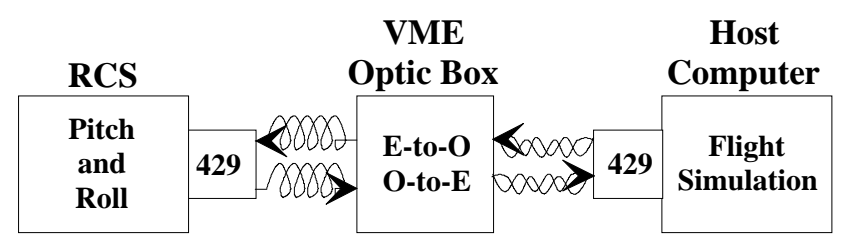

Figure 3. Closed-Loop System.

Two platforms were considered. The first alternative is a VME-based system, Figure 4, where the flight simulation host is a VME-based processor that communicates with the development processor, a PC, via a secondary link, an RS232 or an Ethernet.

VME VME

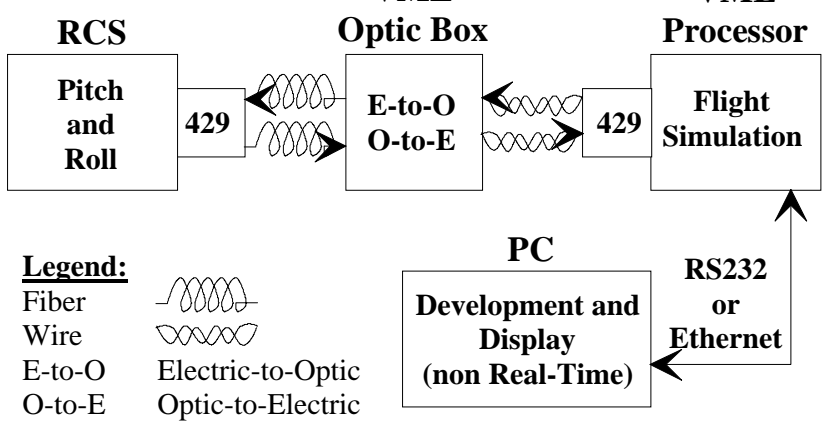

Figure 4. VME-Based System. 
The second alternative bypasses the VME-based processor and implies implementation as well as operation of the flight simulation on one or two PCs, Figure 5.
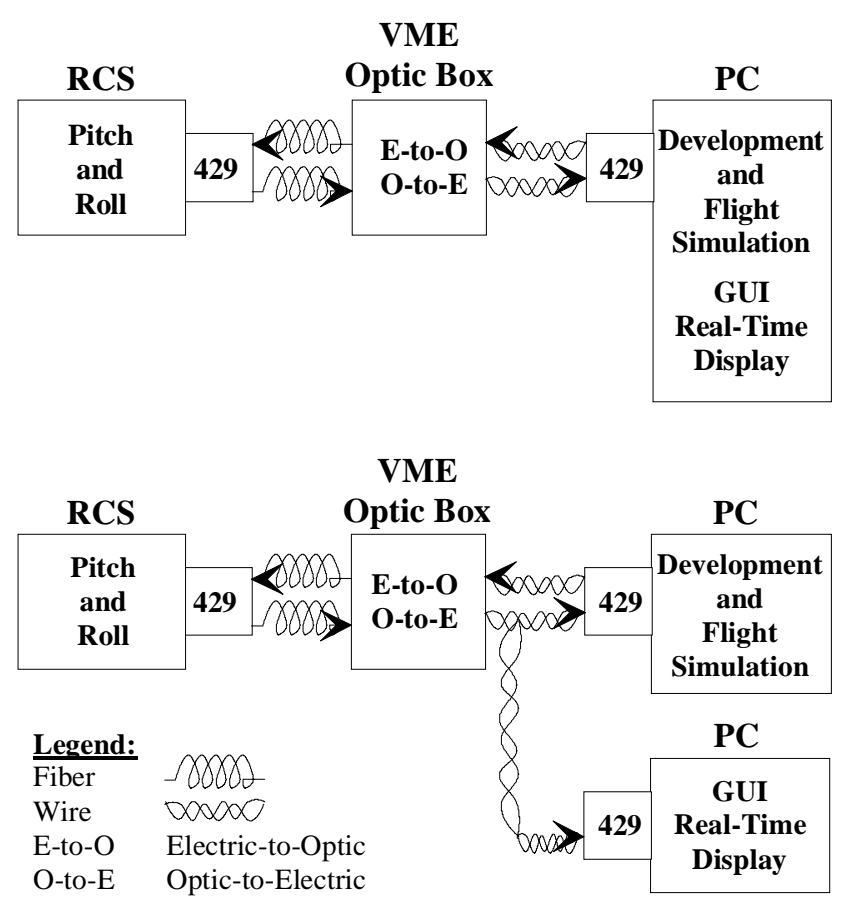

Figure 5. PC-Based Systems.

\section{PC or VME?}

A VME-based system consists of expensive hardware and software compared to a PC-based system. Although there are extensive and specialized software packages available for the VME-based systems, there are steep learning curves associated with them. However, with in-house knowledge of $\mathrm{C} / \mathrm{C}++$ and Windows Development software, the PC-based system imposed only a minimal learning curve. Regardless of the choice of architecture, the only new element that needed to be developed was a 429-interface package.

The VME-based systems were expected to be able to handle real-time operation of the flight simulation. However, real-time display of data would still have to be done through a secondary network and on a PC. As a result, managing the critical timing issues between the two computers was not a trivial task. Since available PCs are very fast and powerful and relatively inexpensive, it seemed practical to attempt to perform the flight simulation operation, as well as collection and display of data on one PC. The challenge, therefore, was proving the feasibility of managing the system and real-time display of data on a PC.

\section{Software Development}

A Graphical User Interface (GUI) package was developed for Windows 95 using $\mathrm{C} / \mathrm{C}++$ languages. This modularized and userfriendly software consists of 429-interface and flight simulation code. It manages communication between the RCS and the flight simulation code and displays the data in realtime while meeting timing requirements of the RCS, Figure 6. This software consists of 11 windows that depict all Closed-Loop System activities. Roll and Aileron command (DELAC), Pitch and Elevator command (DELEC), and Altitude (ALT) and Radio Altitude (HR-ALT) are displayed in three different windows to provide the user with visual correlation between the RCS inputs and their corresponding output commands. The lateral position (YCG), throttle (THROT), and yaw are also displayed separately. In addition to an error monitoring window, there are four other widows that display the discrete values reported by RCS. As a result, this software monitors all activities of this Closed-Loop System. This real-time monitoring capability is essential for testing the system in the presence of RF.

To prevent damaging the RCS, RF and field strength are gradually increased until a region of susceptibility is visually detected. During test runs, the airplane attitude as well as RCS status information, i.e., 429 discrete words, are displayed in real-time in their corresponding windows. Upon upset detection, RF is turned off for the remainder of the flight while system activities are continuously monitored and data 


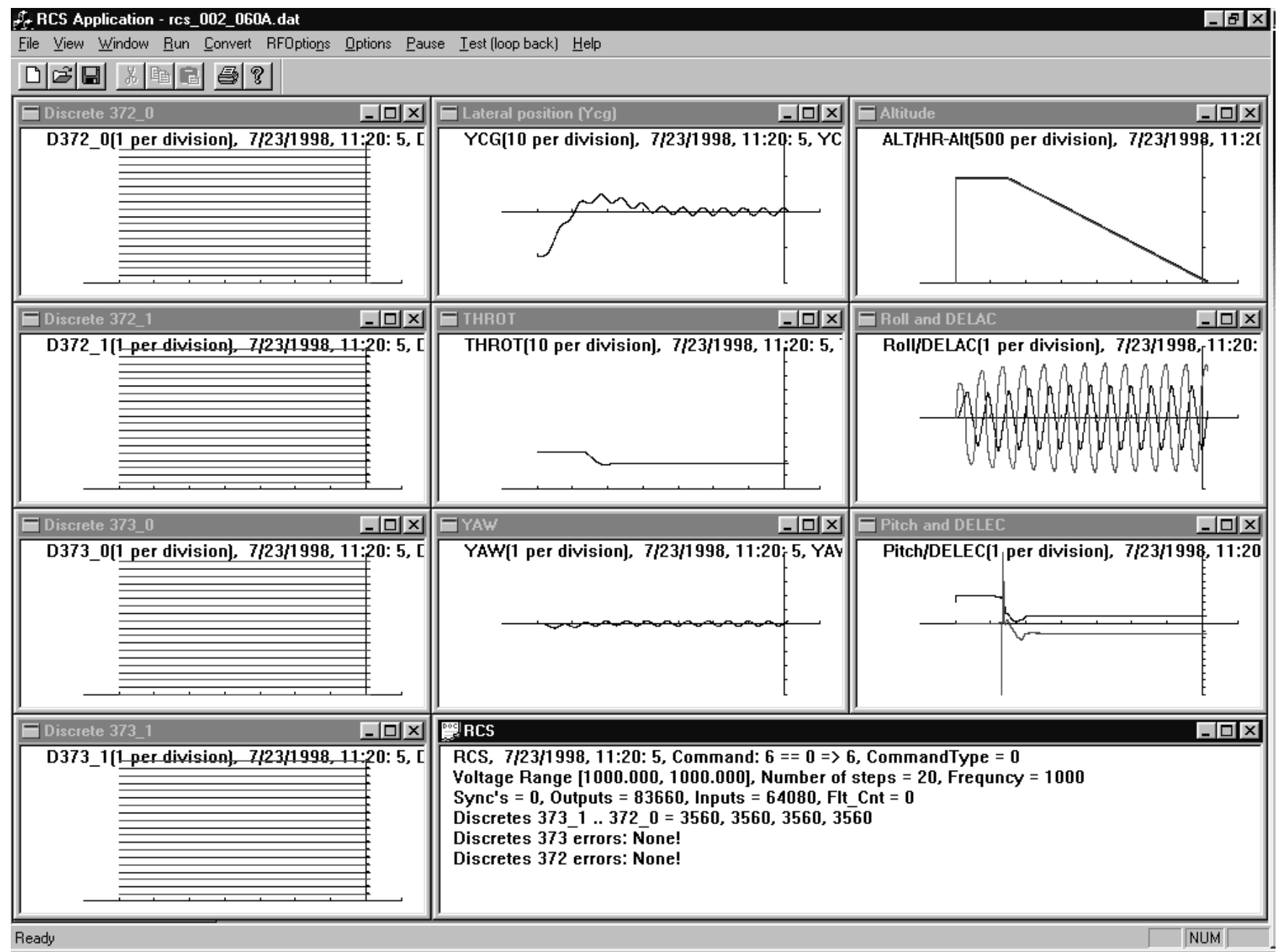

Figure 6. RCS-Win Software.

collected. Through this user-friendly software, test conditions can be specified and recorded along with the data for future references.

\section{Data Management}

The data along with the test conditions such as the RCS control flags, field strength range, and initial frequency are stored on the hard disk. Each file is approximately 1.5 Mbyte. A 1 Gbyte removable hard drive and a CD-ROM writer are used for archival storage. The collected data are in ARINC format [3] in order to minimize the storage requirements. The user interface software is then used to convert these data files to the desired format for post analysis purposes.

\section{Tests}

Tests for the RCS are conducted in two phases. Phase one consists of testing under ideal conditions where EME is absent. Phase two consists of testing in the HIRF chambers in the presence of EME. The specific goals are to characterize the RCS functionality and the RCS upset recovery scheme, to verify control laws 
and flight simulation integrity, and to assess RCS performance under various conditions.

Phase one was necessary in order to resolve discrepancies in the Interface Control Document (ICD) and to debug the system as a whole. Completion of phase one also led to the detection of errors in the implementation of the flight control codes in the RCS, Figure 7.
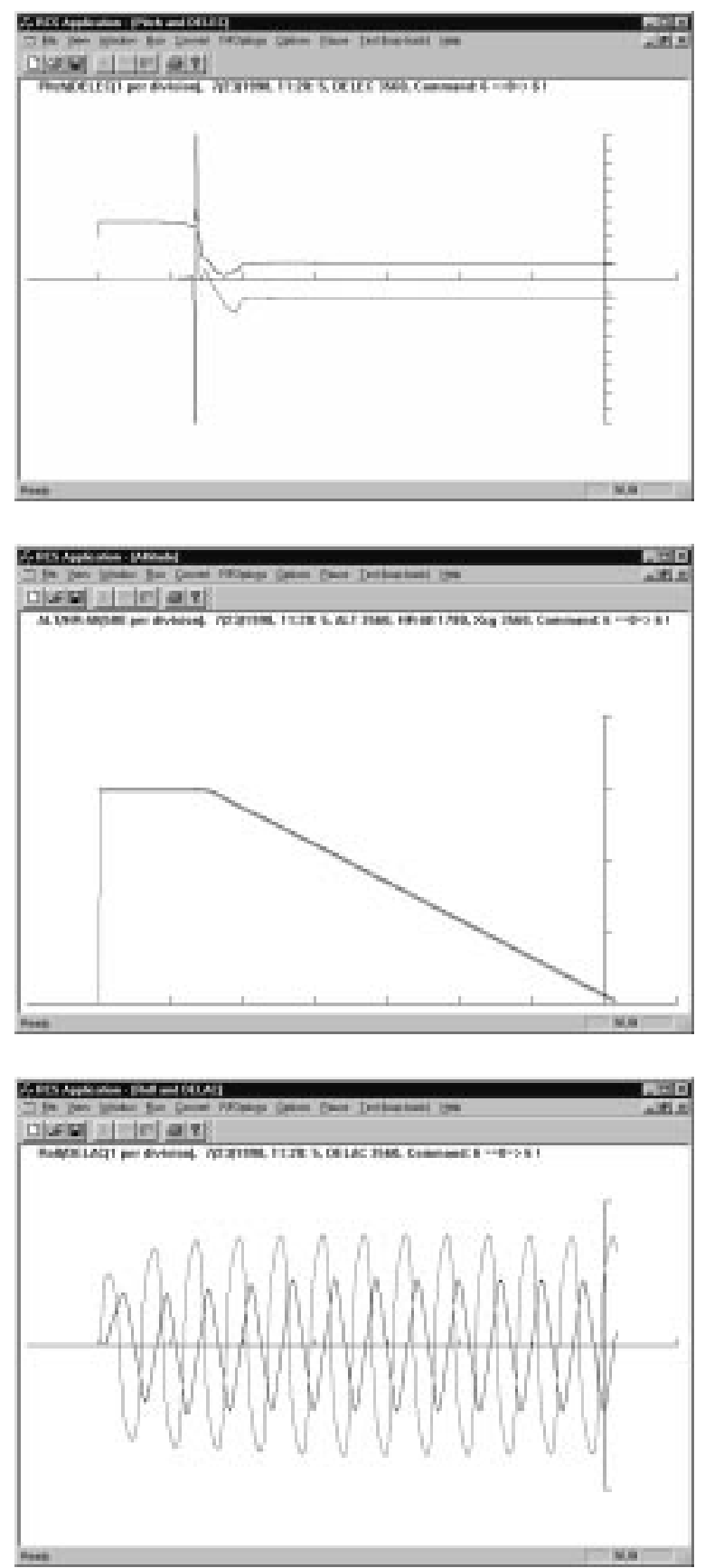

Figure 7. RCS 737 Autoland, no EME.
As shown in the Pitch and DELEC window of Figure 7, there is a spike in the DELEC command at the glide slope engagement as reported by the RCS. Although this discrepancy is not detrimental to the operation of this Closed-Loop System, it is currently being resolved.

In Figure 7, Pitch and Elevator command, Altitude and Radio Altitude, and Roll and Aileron command are displayed in three different windows, respectively, to provide visual correlation between the RCS inputs and their corresponding outputs. The solid lines are for RCS inputs and the dotted lines for RCS outputs.

Phase two of the test is currently in progress and to date, two tests have been completed.

\section{Description of Experiments}

The radiation environment of the HIRF Laboratory is the reverberation chamber. Within the chamber are both transmitting and receiving antennas as well as any sensors that might be needed for a specific test. The components of the signal generation and measurement instrumentation consist of a synthesized sweeper for signal generation, a network analyzer, a spectrum analyzer, an oscilloscope, and a high-power amplifier $[4,5]$. These devices are software controlled from within the HIRF Laboratory. The advantage of performing tests in mode-stirred chambers is that the equipment is subjected to fields at all angles of incidence simultaneously. Therefore, the worst case conditions are represented during the test. The reverberation chambers within the HIRF Laboratory provide near-homogeneous randomized electromagnetic fields and make it possible to place the entire target unit in the test environment.

During the test, the RCS is placed inside one of the mode-stirred chambers of the HIRF Laboratory and is interfaced to the flight 
simulation computer. Electrical isolation is achieved via fiber optic cables.

While testing the RCS in the HIRF chamber, RF and/or field strength are gradually increased until a region of susceptibility is detected. To prevent damaging the RCS and upon upset detection, RF is turned off for the remainder of the test. The test is then resumed with finer increments of frequencies and/or field strength around the susceptibility region.

\section{Closed-Loop HIRF Data}

The RCS was exposed to RF continuously; however the field strength was ramped up from minimum to maximum in 10second intervals while keeping the frequency constant. The same process was repeated for all frequencies. Table 1 is an example of the test conditions.

Table 1. Test Conditions with Continuous HIRF Exposure.

\begin{tabular}{|c|c|c|}
\hline $\begin{array}{c}\text { Frequency } \\
(\mathbf{M H z})\end{array}$ & $\begin{array}{c}\text { Min Field } \\
(\mathbf{V} / \mathbf{m})\end{array}$ & $\begin{array}{c}\text { Max Field } \\
(\mathbf{V} / \mathbf{m})\end{array}$ \\
\hline 200 & 200 & 1000 \\
\hline 250 & 200 & 1000 \\
\hline$\ldots$ & $\ldots$ & $\ldots$ \\
\hline 2000 & 200 & 1000 \\
\hline
\end{tabular}

To date, no repeatable upsets have been detected for the frequencies and power levels shown in Table 1. Besides Continuous Wave (CW), RCS has been exposed to Square Wave (SQW) with all modulations per DO-160 specification for all of the frequency and field strength ranges specified in Table 1 . Preliminary data from 175 flights indicate that the RCS is resilient to the EME.

Upon disabling the recovery capability of the RCS and subjecting the RCS to the same set of tests, RCS seemed somewhat more vulnerable to RF. Figure 8 shows a second spike in the Pitch and DELEC window that is a result of RF induced upset. As evident in the Altitude window, this upset caused the airplane to crash. Out of 165 flights, two upsets were observed, but they were not repeatable.
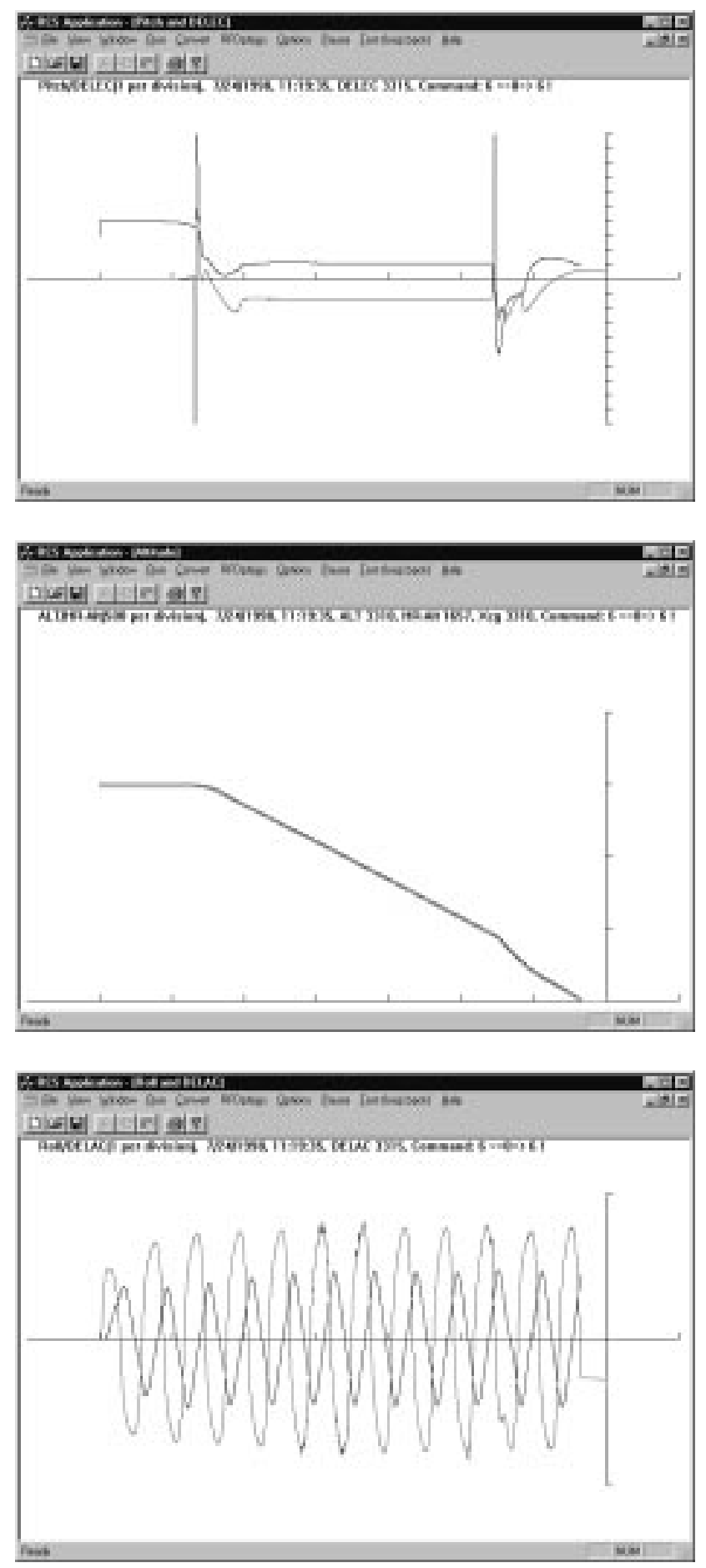

Figure 8. RCS 737 Autoland with Continuous HIRF Exposure and RCS Recovery Scheme Disabled. 


\section{Future Test Plans}

Preliminary test results indicate resiliency of the RCS to the EME at frequencies and power levels used in tests. Since following the DO-160 specification will require considerable amount of time in order to find susceptible frequencies and power levels, another approach will be considered. The next test of RCS will be conducted in the SemiAnechoic chamber of the HIRF Laboratory. In this chamber emission tests are expected to result in detection of some of the susceptibility regions of the RCS. The test will continue in the reverberation chambers to verify and characterize the predicted susceptibility regions. In order to fully characterize the performance capabilities of the RCS to recover from upsets, some of the RF shielding will have to be removed to increase the number of upsets that occur during the tests.

\section{Summary}

The design and development of a Closed-Loop System to study and evaluate the performance of the advanced RCS architecture when subjected to EME is presented. The integration of this hardware proved to be a major undertaking. During this process a few bugs in the 737-control law implementation of the RCS were found that are being resolved. In support of this Closed-Loop System, a Windows-based software package was developed to handle the time critical communication of data and commands between the RCS and flight simulation code, in real-time while meeting the stringent hard deadlines. This package consists of an ARINC429 bus driver, flight simulation code and GUI-based displays of the key elements of the control laws, as well as the airplane attitude. As a result, this package enables the researchers to monitor all activities of the airplane during the flight in real time. The real-time capability of this package is crucial during the EME testing of the hardware.

\section{References}

[1] Celeste M. Belcastro, "Closed-Loop HIRF Experiments Performed on a Fault Tolerant Flight Control Computer," 16th AIAA/IEEE DASC, Irvine, CA, October 1997.

[2] R.F. Hess, "Computing Platform Architectures for Robust Operation in the Presence of Lightning and Other Electromagnetic Threats," 16th AIAA/IEEE DASC, Irvine, CA, October 1997.

[3] ARINC Specification 429P1-15, September 1, 1995.

[4] Daniel M. Koppen, "Open-Loop HIRF Experiments Performed on a Fault Tolerant Flight Control Computer," 16th AIAA/IEEE DASC, Irvine, CA, October 1997.

[5] Rueben A. Williams, "The NASA High Integrity Radiated Fields Laboratory," 16th AIAA/IEEE DASC, Irvine, CA, October 1997. 